\title{
Chemical Vapor Doping of transparent and conductive films of carbon nanotubes
}

Hongjun Gao ${ }^{1,2}$, Ricardo Izquierdo ${ }^{2}$, Vo-Van Truong $*^{1}$

${ }^{1}$ Department of Physics, Concordia University, 7141 Sherbrooke Ouest, Montréal, Québec H4B 1R6, Canada, ${ }^{2}$ Département d'informatique, Université du Québec à Montreal (UQAM), Case postale 8888, succursale Centre-ville, Montréal, Québec H3C 3P8, Canada

Key words: chemical vapor doping (CVDo), transparent and conductive films (TCF), carbon nanotubes (CNT), networks, acid treatment

\section{Abstract}

An alternative Chemical Vapor Doping (CVDo) method for doping transparent and conductive films of carbon nanotubes (CNTs) is presented. The different effects between the traditional solution doping process and the proposed vapor doping method are compared in detail by means of optical microscopy, SEM, sheet resistance measurements, and Raman and UV-Vis-NIR spectroscopy. It was shown that the level of reduced sheet resistance was comparable for the two treatments but the vapor-treated film displayed better stability in air as well as other significant advantages that include its appropriateness for different types of flexible substrates. A mechanism for chemical vapor doping is proposed. 


\section{Introduction}

Conductive films of carbon nanotubes (CNTs) are very promising materials as transparent electrodes because of their high conductivity, high transparency, flexibility and low cost of fabrication compared to traditional materials like indium tin oxide (ITO). CNT transparent and conductive films (TCFs) have a large potential of use in touch screens, flat panel displays, thin film solar cells, organic light emitting diodes, electrochromic devices, electrostatic shielding and more. The quality of the film depends on the type of CNTs, the dispersion conditions, the fabrication process and the post treatment. Lots of work has been done to improve the film's conductivity and transparency [1-3]. In these studies, doping of CNT films is one of the main concerns and thought to be essential for performance improvement and for the future applications. There are several possible methods for doping CNT films: encapsulation in the interior spacing, intercalation of electron donors or acceptors, sidewall adsorption and covalent functionalization, etc. Normally, non-covalent doping has a lower binding energy and it is less stable but has the preferred capacity to increase the charge carrier mobility in most cases. Typical dopants can be categorised into p-type such as strong acid $\left(\mathrm{HNO}_{3}\right)$ [4], oxidizing gas $\left(\mathrm{NO}_{2}[5], \mathrm{O}_{2}[6], \mathrm{Br}_{2}[7]\right)$, other aggressive chemicals( $\left.\mathrm{SOCl}_{2}[8], \mathrm{F}_{4} \mathrm{TCNQ}[9]\right)$ and n-type such as K[7]. The Brønsted acid $\mathrm{HNO}_{3}$ as an ordinary chemical agent is easily accessible and has been used for the purification and post-treatment of CNTs since their discovery. In recent years, there has been an increase of interest in $\mathrm{HNO}_{3}$ treatment on CNT films for doping because of its simplicity and efficacy [10-19]. The traditional way of doping CNT with $\mathrm{HNO}_{3}$ is by immersing the films in the acid solution. However, this method introduces limitations on the substrates that can be used for depositing the CNT films. Different points of view on the mechanism have been stated in these studies. It has been reported that SWCNT films treated in saturated nitric acid vapor overnight, show graphite-like intercalation behaviour and the consequent hole doping effect [16]. However, a long-time of treatment (12 hours) was used and there were no details on 
the causes of the improvement of film conductivity. Here we present an alternative Chemical Vapor Doping (CVDo) method for the treatment of CNT films. This method supplied an alternative proof to help us elucidate the mechanism of the improvement of the film conductivity.

\section{Experiment}

\section{Materials}

We used P2-SWNT from Carbon Solutions, Inc. [20]. These CNTs were synthesized by using the electric arc discharge method with $\mathrm{Ni} / \mathrm{Y}$ catalysts then they were purified by air oxidation and subsequently treated to remove the catalyst. According to the supplier, the purified material closely approximates the pristine state with low functionality and low chemical doping. The ratio of semiconducting to metallic SWNTs produced by this method is 2 to 1 . These CNTs have a bundle length of $500 \mathrm{~nm}$ to $1.5 \mu \mathrm{m}$ and a bundle diameter of 4 to $5 \mathrm{~nm}$ in the powder form. From their analysis, the individual tubes have a narrow diameter distribution peaking at $1.4 \mathrm{~nm}$. The SWNTs can be dispersed in DMF (Dimethylformamide) at concentrations up to $0.1 \mathrm{mg} / \mathrm{ml}$; they can also be dispersed in water by using surfactants such as Sodium dodecyl sulfate (SDS).

\section{Film preparation and treatment}

The transparent films of carbon nanotubes were fabricated by using the vacuum filtration method [21]. Firstly, 10mg P2-SWNT and 0.4g Sodium dodecyl sulfate (SDS) were mixed with $40 \mathrm{ml}$ de-ionized water. Then the mixture was ultrasonicated in a Cole-Parmer ultrasonic machine model 08890-11 with $155 \mathrm{~W}$ power for 48 hours to assure good dispersion. Thirdly, the solution was centrifuged for one hour at 30000 rpm in a Beckman L8-70M centrifuge equipped with a SW41Ti rotor and only the supernatant was kept for next step. Fourthly, the dispersion of CNT was filtered through a $0.1 \mu \mathrm{m}$ pore size and $47 \mathrm{~mm}$-diameter mixed cellulose membrane from 
Millipore. After naturally drying on top of the filter for 24 hours in a clean room, the CNT film was transferred onto a glass substrate by dissolving the cellulose membrane with acetone. To compare the effects of different treatment process, we cut the same film into three parts for the following steps. The three parts are named the original, $\mathrm{HNO}_{3}$ vapor and solution treated $\mathrm{CNT}$ films respectively. For the $\mathrm{HNO}_{3}$ solution treatment process, we used $68-70 \%$ ACS grade $\mathrm{HNO}_{3}(16 \mathrm{M})$. The sample was put into the solution directly and kept there for one hour. Then it is taken out and dried in air. For the $\mathrm{HNO}_{3}$ vapor treatment process, the sample was put in a closed glass chamber about $1 \mathrm{~cm}$ above the concentrated $\mathrm{HNO}_{3}$ solution for one hour. All these treatments were done in the clean room conditions.

\section{Measurement and analysis}

For optical microscope imaging, we used a Nikon Eclipse LV 150 microscope equipped with a Nikon D5000 camera. A field emission scanning electron microscope (FE-SEM) Hitachi S-4700 was used for the surface morphology characterization, with a $3 \mathrm{kV}$ acceleration voltage. We used a FT-Raman Spectrometer (Model: Thermo Scientific, Nicolet 6700 / NXR FT-Raman Module) for the Raman spectroscopy of the CNT film with a laser power of $0.24 \mathrm{~W}$ and a wavelength of $976 \mathrm{~nm}$. A spectrophotometer Cary 5000 UV-Vis-NIR was used to record the transmittance spectroscopy in the range from $300 \mathrm{~nm}$ to $3300 \mathrm{~nm}$. For the four-point probe measurement of sheet resistance $\left(\mathrm{R}_{\mathrm{S}}\right)$, we used a LUCAS LABS 302 probe station equipped with a KEITHLEY 6220 Precision Current Source and a KEITHLEY 2000 multimeter.

\section{Results and Discussion}

The CNT films treated by solution and vapor processes showed different surface morphology. We examined this by optical microscope and SEM. Figure 1 shows the optical image of a CNT film deposited on glass substrate of which the bottom half 
was immersed in $\mathrm{HNO}_{3}$ solution (part a in Figure 1) while the top half was exposed to the vapor phase (part b in Figure 1). It is shown in the figure that the immersed part looks a little darker under the optical microscope than the part exposed to air. We also observed that when the immersion time was too long, the adhesion between the CNT film and glass substrate could be reduced. This causes wrinkles when the sample is being taken out from the solution. The SEM images of the CNT films treated in $\mathrm{HNO}_{3}$ vapor and solution are presented in Figures 2 and 3 respectively. In the SEM images, the CNT film treated in $\mathrm{HNO}_{3}$ vapor has lower tube density and more particles on the film surface than the CNT film treated in $\mathrm{HNO}_{3}$ solution. On the contrary, the CNT film treated in $\mathrm{HNO}_{3}$ solution looks denser and has fewer particles on the film surface. This means that in the solution process, the liquid washed off some adsorbates with low adhesion to the film surface, causing the nanotubes to follow the direction of the liquid's flow when the sample was taken out from the solution and helping them to aggregate vertically on the surface to make the film thinner in the drying process.

The UV-Vis-NIR optical transmittance spectra of the CNT films before and after $\mathrm{HNO}_{3}$ vapour and solution treatments are shown in Figure 4. For the original, $\mathrm{HNO}_{3}$ solution and vapor treated samples, the transmittance at $550 \mathrm{~nm}$ is of $72.0 \%, 72.2 \%$ and $71.0 \%$ respectively. From these optical transmittance spectra measurements, we can see that the differences are negligible even in the range of $400-800 \mathrm{~nm}$. This is comparable for the requirement of transparency in the visual wavelength region. While for the absorption intensity of the interband energetic transition $\mathrm{S}_{11}$, there are significant drops after acid treatments. This evidence indicates hole doping [15,22]. Compared with $\mathrm{HNO}_{3}$ solution doping process, after the $\mathrm{HNO}_{3}$ vapor process over the same time period, the $S_{11}$ absorption peak is almost bleached, which indicates a higher degree of doping in this case. Figure 4 also shows that there is a smaller reduction of $\mathrm{S}_{22}$ peak for the sample treated in vapor as compared with the sample treated in solution. The second semiconducting transition $\mathrm{S}_{22}$ is less sensitive to doping but is useful for the determination of the relative purity of SWCNTs [20]. From our results, the slightly decreased $\mathrm{S}_{22}$ absorption of $\mathrm{HNO}_{3}$ vapor treated samples might indicate an 
increase in carbonaceous impurities after the doping process.

The Raman spectra of the original, $\mathrm{HNO}_{3}$ vapor and solution treated $\mathrm{CNT}$ films are shown in Figure 5. They have the typical peaks of radial breathing mode (RBM), D-band and G-band for SWCNTs. The G-band can be used to probe charge transfer from doping, to distinguish metallic and semiconducting tubes through their strong differences in line shapes [23]. There were studies on the effect of electrochemical pand n-doping of SWNT films investigated by in situ resonance Raman spectroscopy [11]. Also, it has been revealed that in the process of doping CNT, for typical electron-donor (potassium, K) and electron-acceptor (bromine, $\mathrm{Br}_{2}$ ) dopants, the high-frequency tangential vibrational modes of the carbon atoms shift to lower (for K) or higher (for $\mathrm{Br}_{2}$ ) frequencies respectively [24]. From Figure 5, we know the following for all the CNTs: they are single walled; there is a metallic percentage from the Breit-Wigner-Fano (BWF) line shape; there is little or no amorphous carbon impurity from the low D-band. For original, vapor and solution treated samples, we observed that they had a different G-band which was $1592.91 \mathrm{~cm}-1,1593.63 \mathrm{~cm}-1$ and $1596.03 \mathrm{~cm}-1$ respectively. This upshift of G-band is associated with the removal of electrons from the SWNT $\pi$-band and indicates a p-doping effect on the film by the $\mathrm{HNO}_{3}$ treatments. Also, there is a slight increase of BWF line intensity, which indicates the increase in plasmon coupling between tubes and the improvement of the film metallicity after treatment. From the above analysis, we got proof of charge transfer from Raman spectroscopy. By the way, there is some evidence supporting the hole-doping mechanism of the SWCNTs by the $\mathrm{HNO}_{3}$ solution treatment in the previous report because of the down shift of $\mathrm{C} 1 \mathrm{~s}$ core level peak in the X-ray photoelectron spectroscopy (XPS) measurements [4].

The measurements of sheet resistance $\left(\mathrm{R}_{\mathrm{s}}\right)$ for the original, $\mathrm{HNO}_{3}$ vapor and solution treated CNT films are shown in Figure 6. When a film was deposited on filter, it was cut into three parts and then transferred to glass substrate. We measured the sheet resistance just after the films were transferred, which was marked as the day " 0 " in 
Figure 6. After one day, we kept one sample without treatment for comparison, while the other two were used for HNO3 solution and vapor treatment respectively. The results of the first day represented the sheet resistance measured just after the acid treatments. For both of the solution and vapour treatment processes, the reduction of sheet resistance by a factor of 4 was achieved just after the acid treatment, compared with 2.5 times in [13]. There was a greater improvement of electrical conductivity observed in the semiconducting SWNT film after chemical treatment as compared to the case of metallic SWNT films [15]. Our sample has a high semiconducting to metallic ratio which is $2: 1$ and we used a $16 \mathrm{M} \mathrm{HNO}_{3}$ which is more concentrated. These factors contribute to the higher reduction of sheet resistance. Also, we measured the changes of sheet resistance with time for three weeks. It is shown that $\mathrm{R}_{\mathrm{s}}$ increased with time and became stable in two weeks. For the vapour treated sample, the $\mathrm{R}_{\mathrm{s}}$ increased to two thirds of its original value while for the solution treated sample, the $\mathrm{R}_{\mathrm{s}}$ almost recovered to its original value.

This special vapor treatment process helped us to understand the role of SDS in the improvement of film conductivity. In the dispersion process for preparing the CNTs solution, lots of SDS will adsorb on the individual CNT surface, wrap the CNT, form a monolayer and eventually separate the CNTs bundle. SDS is a surfactant molecule with long chain. They have good adhesion force on the outer surface of CNTs. SDS remains to be a main residue on CNT films surface after the filtration in the fabrication process. For the $\mathrm{HNO}_{3}$ solution treated sample, most SDS molecules were washed off by the $\mathrm{HNO}_{3}$ solution treatment process. Several previous studies on $\mathrm{HNO}_{3}$ treatment stated that the improvement of film conductivity was attributed to the removal of SDS molecules $[13,14,19]$. In our $\mathrm{HNO}_{3}$ vapor treatment process, there is no washing step involved. We know that in this circumstance, SDS is not removed by the process itself. However, we got a reduction of sheet resistance with the same level as in the $\mathrm{HNO}_{3}$ solution treatment process. From the analysis above, we can conclude that the complete removing of SDS from the CNT film does not play the most important role for the improvement of the conductivity of the CNT film. Nevertheless, 
for the $\mathrm{HNO}_{3}$ vapor treated sample, there could be some displacement of SDS among the CNTs. Thus the cross junctions between CNTs are improved.

In our case, the chemical doping of $\mathrm{HNO}_{3}$ has an obvious effect. As a strong Brønsted acid, $\mathrm{HNO}_{3}$ can be intercalated reversibly in the expanded lattice of the SWCNT bundles, which is supported by X-ray diffraction evidence [25]. On the one hand, nitric acid forms an azeotrope with water at a concentration of $68 \%$. Nitric acid is subject to thermal or light decomposition:

$$
4 \mathrm{HNO}_{3} \rightarrow 2 \mathrm{H}_{2} \mathrm{O}+4 \mathrm{NO}_{2}+\mathrm{O}_{2}
$$

The nitrogen oxides produced dissolve partly or completely in the acid. $\mathrm{NO}_{2}$ can adsorb in an asymmetric configuration via at least one of the oxygen atoms by interacting with multiple nanotubes within a bundle of SWNTs [26]. On the other hand, since nitric acid has both acidic and basic properties, it can undergo an autoprotolysis reaction:

$$
2 \mathrm{HNO}_{3} \leftrightarrow \mathrm{NO}_{2}^{+}+\mathrm{NO}_{3}^{-}+\mathrm{H}_{2} \mathrm{O}
$$

The hypothesis that after the $\mathrm{HNO}_{3}$ purification process, the materials were left as the salts of the form $\left(\mathrm{SWNT}^{\mathrm{n}+}\right)\left(\mathrm{HNO}_{3}\right)_{\mathrm{m}}\left(\mathrm{NO}_{3}{ }^{-}\right)_{\mathrm{n}}$ was supported by a transmission spectroscopy analysis. The trapped $\mathrm{NO}_{3}{ }^{-}$counter ions can be removed after heating in vacuum[27].

Here we propose a "Piercing Mechanism" in this doping process. In the $\mathrm{HNO}_{3}$ solution, there is plenty of $\mathrm{NO}_{3}^{-}$. In vapor phase, there are more $\mathrm{NO}_{2}$. Since $\mathrm{NO}_{3}{ }^{-}$or $\mathrm{NO}_{2}$ is much smaller than SDS and nanotubes, it will pierce most easily into the interface between the surfactants and nanotubes. Then SDS will be excluded from the direct contact with nanotubes. CNTs are amphoteric and possess the ability to accept (or donate) electrons from (or to) dopant atoms or molecules. It is assumed that the oxidizing reagents $\mathrm{NO}_{3}^{-}$and $\mathrm{NO}_{2}$ which tend to form stable planar resonating electronic structure originated from $\mathrm{sp}^{2}$ hybridization and are also known to form electron withdrawing groups with high electronegativity will work as electron acceptors when they are in contact with CNTs. So the hole doping effects happen and 
contribute to the improvement of film conductivity. The exclusion of SDS from nanotubes will help individual nanotubes to contact each other directly and form new intertube junctions. Normally, it takes a long-time ultrasonication process to separate the CNT bundles and the dispersed CNT solution has a tendency to aggregate over time to form bundles. These phenomena signify that the van der Waals forces among tubes are stronger than the forces between SDS and nanotubes. Nanotubes liberated from SDS will then re-form new bundles along part of their length with their near neighbours. In the HNO3 solution treatment and drying process, the film can be densified by the surface tension from the liquid. The phenomenon became more remarkable. This kind of re-bundling and reconnection effect among nanotubes also contributes to the improvement of film conductivity.

The small molecules adsorbed on the nanotubes have a tendency to escape and desorb from the bulk. So the doping effect caused by $\mathrm{NO}_{3}{ }^{-}$or $\mathrm{NO}_{2}$ is not stable. In fact, most of the previous reports about chemical doping showed that the results changed with time [3] and even changed with deionized water washing [19]. In contrast the re-bundling between the nanotubes has a relatively steady effect and will not easily change with time. In a previous study, the low-concentration $\mathrm{HNO}_{3}$ solution as $12 \mathrm{M}$ was used. They got a steady reduction of sheet resistance which was 2.5 times lower than the initial value and showed no proof of doping effect [13]. In our case, we used highly concentrated $\mathrm{HNO}_{3}$ as $16 \mathrm{M}$ and the samples were kept in the dopants for one hour. So the reconnection between the nanotubes was established and the doping effect was assured. Eventually we got a reduction of sheet resistance 4 times lower than the original value but the contribution of chemical doping will decrease with time. This is shown in Figure 6, where $\mathrm{R}_{\mathrm{s}}$ increased with time and stabilized at two thirds of the original value for the vapour treated sample. An interesting effect in the vapor treated sample is that it is more stable in the natural de-doping process and the recovered $R_{s}$ is lower than that of the solution treated sample. This phenomenon could result from the protection effect of SDS. In the vapor treatment process, the SDS is not washed off. It covers part of the outer shell of whole film as a monolayer. Because 
of its strong affinity with nanotubes, the desorption of dopants is thus hindered by the presence of the protecting SDS layer. This effect is similar to the improved stability of transparent $\mathrm{CNT}$ thin films after $\mathrm{HNO}_{3}$ and $\mathrm{SOCl}_{2}$ treatment with a top coating of PEDOT-PSS [28].

In summary, we attributed the improvement of CNT film conductivity to chemical doping by $\mathrm{HNO}_{3}$, exclusion of the SDS between nanotubes and the following re-bundling and reconnection effect. When the reconnection between the tubes is established, the remained SDS molecules on the film surface would protect the film from de-doping and thus have a positive effect on film conductivity.

To verify the doping effect of $\mathrm{HNO}_{3}$ vapor treatment on plastic substrate, we transferred a $37 \mathrm{~mm}$ diameter CNT film onto the transparent polycarbonate substrate. The original sheet resistance is $102.4 \mathrm{ohm} / \mathrm{sq}(\mathrm{T}=47.9 \%$ at $550 \mathrm{~nm})$. After one hour's treatment in $\mathrm{HNO}_{3}$ vapor, the initial sheet resistance was of $23.3 \mathrm{ohm} / \mathrm{sq}$. From figure 7, the transparent polycarbonate substrate showed good chemical compatibility with the $\mathrm{HNO}_{3}$ vapor. It looked as transparent and shiny as before. There was no swelling, no damage or any other obvious changes found on the surface of the polycarbonate substrate. Also, we measured the evolution of sheet resistance over time after $\mathrm{HNO}_{3}$ vapor treatment, as shown in figure 8 . The sheet resistance stabilized at half of the original value after two weeks for the sample being stored in a clean room.

The $\mathrm{HNO}_{3}$ vapor treatment process is very effective and possesses many advantages. Firstly, it is simple compared with solution immersion. Only a small amount of $\mathrm{HNO}_{3}$ solution is enough to produce a large volume of vapor for the treatment. Secondly, it avoids direct contact between the sample and solution. It is good for the samples which are not suitable to be in contact with liquid. We found that the CNT film treated in $\mathrm{HNO}_{3}$ solution easily acquired wrinkles and other damages. Especially, the present process is good for flexible plastic substrates such as polycarbonate with low chemical resistivity to strong acids. Also, it offers the opportunity to conserve 
chemicals in the CNT film which are good additives and should be kept there. Thirdly, there is no contamination to the solution for treatment. Thus the $\mathrm{HNO}_{3}$ solution in the vapor treatment process can be used many times. This makes the process more economical, productive and scalable in industrial application. Fourthly, the vapor treatment process avoids the liquid drying process which leaves traces of solute from the natural non-uniform evaporation. Fifthly, as to the doping effect, the vapor treatment demonstrated larger $\mathrm{R}_{\mathrm{s}}$ reduction for the same process time. Meanwhile, the sample with vapor treatment showed better $R_{s}$ stability with time after exposure to air.

\section{Conclusion}

In this paper we presented a $\mathrm{HNO}_{3}$ treatment technique as chemical vapor doping (CVDo) to improve the conductivity of CNT films. It is simple, effective and advantageous as compared to traditional solution treatments. The different results between the solution and vapor doping methods were examined in detail by means of optical microscopy, SEM, sheet resistance measurements, and Raman and UV-Vis-NIR spectroscopy. We proposed a "piercing mechanism" for the doping process and indicated that re-bundling of the dispersed CNTs in the film and the protection effect of SDS molecules were two important factors for the stable reduction of its sheet resistance, while the total removal of SDS in previous reports was not indispensible. We investigated the influence of nitric acid on the electronic properties of single-walled carbon nanotubes (SWCNTs). This doping effect was characterized by the blue shift of the G-band in Raman spectroscopy and the reduction of the $\mathrm{S}_{11}$ line in the UV-Vis-NIR absorption measurement. Thus, this $\mathrm{HNO}_{3}$ chemical vapor doping (CVDo) method has been shown to be most efficient. Because of its significant advantages, it has the potential to greatly facilitate the wide-spread and increasing application of transparent and conductive films (TCFs) of carbon nanotubes (CNTs) both in laboratories and in industries. 


\section{Acknowledgements}

This work was supported by the Natural Science and Engineering Research Council of Canada (NSERC).

\section{References}

[1] R.H. Baughman, A.A. Zakhidov, W.A. de Heer, Science 297 (2002) 787.

[2] L.B. Hu, D.S. Hecht, G. Gruner, Chem. Rev. 110 (2010) 5790.

[3] D.S. Hecht, L.B. Hu, G. Irvin, Adv. Mater. 23 (2011) 1482.

[4] R. Graupner, J. Abraham, A. Vencelova, T. Seyller, F. Hennrich, M.M. Kappes, A. Hirsch, L. Ley, Phys. Chem. Chem. Phys. 5 (2003) 5472.

[5] J. Kong, N.R. Franklin, C.W. Zhou, M.G. Chapline, S. Peng, K.J. Cho, H.J. Dai, Science 287 (2000) 622 .

[6] D. Kang, N. Park, J.H. Ko, E. Bae, W. Park, Nanotechnology 16 (2005) 1048.

[7] R.S. Lee, H.J. Kim, J.E. Fischer, A. Thess, R.E. Smalley, Nature 388 (1997) 255.

[8] U. Dettlaff-Weglikowska, V. Skakalova, R. Graupner, S.H. Jhang, B.H. Kim, H.J. Lee, L. Ley, Y.W. Park, S. Berber, D. Tomanek, S. Roth, J. Am. Chem. Soc. 127 (2005) 5125.

[9] T. Takenobu, T. Kanbara, N. Akima, T. Takahashi, M. Shiraishi, K. Tsukagoshi, H. Kataura, Y. Aoyagi, Y. Iwasa, Adv. Mater. 17 (2005) 2430.

[10] F. Hennrich, R. Wellmann, S. Malik, S. Lebedkin, M.M. Kappes, Phys. Chem. Chem. Phys. 5 (2003) 178.

[11] P. Corio, A. Jorio, N. Demir, M.S. Dresselhaus, Chem. Phys. Lett. 392 (2004) 396.

[12] X.F. Zhang, T.V. Sreekumar, T. Liu, S. Kumar, J. Phys. Chem. B 108 (2004) 16435.

[13] H.Z. Geng, K.K. Kim, K.P. So, Y.S. Lee, Y. Chang, Y.H. Lee, J. Am. Chem. Soc. 129 (2007) 7758.

[14] S.B. Yang, B.S. Kong, J. Geng, H.T. Jung, J. Phys. Chem. C 113 (2009) 13658.

[15] R.K. Jackson, A. Munro, K. Nebesny, N. Armstrong, S. Graham, Acs Nano 4 (2010) 1377.

[16] K. Kamaras, A. Pekker, B. Botka, H. Hu, S. Niyogi, M.E. Itkis, R.C. Haddon, Phys. Status Solidi B 247 (2010) 2754.

[17] Y.L. Zhao, W.Z. Li, Microelectron. Eng. 87 (2010) 576.

[18] E.H. Lee, J.H. Ryu, J. Jang, K.C. Park, Jpn. J. Appl. Phys. 50 (2011).

[19] G.H. Xu, J.Q. Huang, Q. Zhang, M.Q. Zhao, F. Wei, Appl. Phys. a-Mater. 103 (2011) 403.

[20] M.E. Itkis, D.E. Perea, S. Niyogi, S.M. Rickard, M.A. Hamon, B. Zhao, R.C. Haddon, Nano Lett. 3 (2003) 309.

[21] Z.C. Wu, Z.H. Chen, X. Du, J.M. Logan, J. Sippel, M. Nikolou, K. Kamaras, J.R. Reynolds, D.B. Tanner, A.F. Hebard, A.G. Rinzler, Science 305 (2004) 1273.

[22] S. Kazaoui, N. Minami, R. Jacquemin, H. Kataura, Y. Achiba, Phys. Rev. B 60 (1999) 13339.

[23] M.S. Dresselhaus, G. Dresselhaus, R. Saito, A. Jorio, Phys. Rep. 409 (2005) 47.

[24] A.M. Rao, P.C. Eklund, S. Bandow, A. Thess, R.E. Smalley, Nature 388 (1997) 257.

[25] C. Bower, A. Kleinhammes, Y. Wu, O. Zhou, Chem. Phys. Lett. 288 (1998) 481.

[26] H. Chang, J.D. Lee, S.M. Lee, Y.H. Lee, Appl. Phys. Lett. 79 (2001) 3863. 
[27] M.E. Itkis, S. Niyogi, M.E. Meng, M.A. Hamon, H. Hu, R.C. Haddon, Nano Lett. 2 (2002) 155 .

[28] R. Jackson, B. Domercq, R. Jain, B. Kippelen, S. Graham, Adv. Funct. Mater. 18 (2008) 2548. 